\title{
Extra Solar Planets in Habitable Zone: The Role of Chaos Kountouris George*
}

Hellenic Amateur Astronomy Association, Sector of Astrophysics and Cosmology, Greece

*Corresponding author: George K, Hellenic Amateur Astronomy Association, Sector of Astrophysics and cosmology, Greece, Tel: +30 6981-762 034; E-mail: koungeo8@otenet.gr

Received date: January 2, 2018; Accepted date: January 18, 2018; Published date: January 22, 2018;

Copyright: @ 2018 George K. This is an open-access article distributed under the terms of the Creative Commons Attribution License, which permits unrestricted use, distribution, and reproduction in any medium, provided the original author and source are credited.

\begin{abstract}
It is generally known, the $20^{\text {th }}$ century is entitled as the century of great discoveries in physics. These discoveries gave answers to a huge number of questions, which, in the previous centuries were belonging to the realm of prejudice, superstitions and, above all, religions.

The bottom line of the above discoveries, which are the General Theory of Relativity, interprets Macro-World issues, and Quantum Mechanics interprets issues of the Micro-Subatomic World. Both of them established the standard model of how the cosmos is working. This standard model still has gaps of unanswered questions, which science in the $21^{\text {st }}$ century, is still working to discover thus, providing some answers. (The major gap is the lack of compatibility between G.T.R. and Q.M., which is interpreting the respective lack of the nature between macro and micro world). In macro world the gaps are related in every sense of celestial bodies what means from planets, extending in stars, galaxies, clusters and superclusters of galaxies up to the entire visible and invisible universe. Our above title constitutes one of these gaps.
\end{abstract}

Keywords: Exoplanet; Asteroid belt; Habitable zone; Super Earths

\section{Introduction}

At the end of the $20^{\text {th }}$ century and specifically in 1995 came a breakthrough, which has to do with the big question from the ancient years of Humanity "if we are alone" on this vast and endless Universe.

The first extra solar planet was discovered, orbiting another star beyond our Sun. In particular on star 51 Pegasi, by the method of radial velocity of star's wobble, which was caused by the presence of a planet with a mass the half of our Jupiter's.

From then until today the number of confirmed exoplanets has so far reached 3,472, orbiting 2,588 stars. The numbers keeps on rising.

After this extraordinary rate of exoplanets detection, the branch of planetary science was subdivided with a new separate branch of extra solar planets science, with the thought that the existence of planets among stars in our galaxy, and in other galaxies is an ordinary phenomenon and not a prerogative of our Sun only.

Essentially this fact has placed a little bit attention on the eternal question of Humanity if we are alone in the Cosmos [1].

Within this relatively short time lapse the Scientific Society organized its research with the goal to detect exoplanets, which are maintaining environmental conditions as similar as possible to those on Earth. In principle they classified the detecting exoplanets based on the planets of our own Solar System. That is:

Jupiters and larger with mass $>6$ Mass of up to the class of Brown Dwarfs;

Neptunes, mini Neptunes with masses 2-6 Mass of;

Rocky planets, Earths, super Earths with masses $<1,25-2$ Mass of;

Where Mass of $=$ Earth's mass.

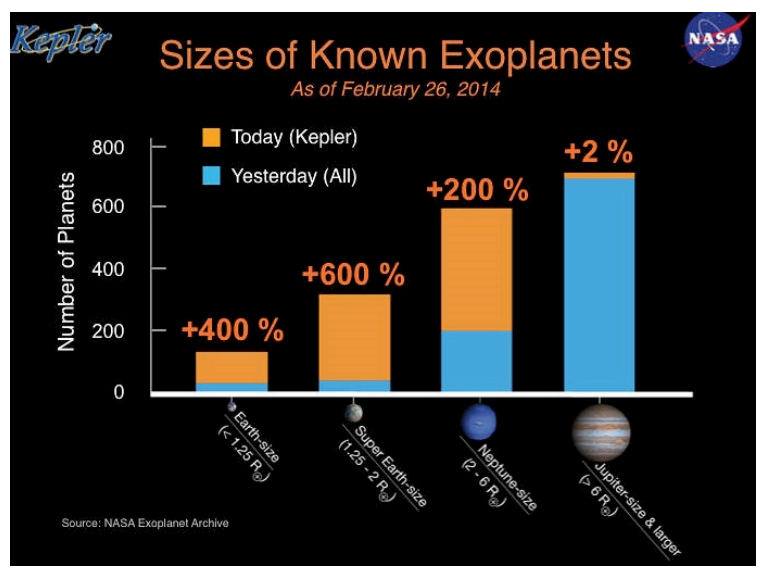

Figure 1: On the rate of increasing exoplanets detection the dominant type is Super Earths.

In Figure 1, we can see the rate (percentage) of rocky planets detection via the valuable assistance of Kepler space observatory. Using this diagram we can say that it has put another little bit, forming the question maybe we are not alone- but who knows?

Based upon Earth's environmental conditions and according to stars spectral types, circular areas around the exoplanets' parent-stars, were established where the planets inside these areas are able to maintain water in its liquid form. These areas were named habitable zones. Figure 1 shows representative three types of all spectral types of stars (O, B, A, F, G, K.M.) where the areas of three zones are shown. The green zone is the habitable zone. The red one is the hot and the blue one is the cold zone. In hotter stars (higher spectral types) the 3 zones are extended farther away than in cooler stars, as our Sun and lower spectral types where they are closer to the star. Also these three zones 
Page 2 of 6

are representing the conditions of water, if it exists on the planet. About the habitable green zone we have talked already. In the blue cold zone water is in the condition of ice. And in the hot red zone in vapors if it has not scattered in space due to the planets' parent star activity.

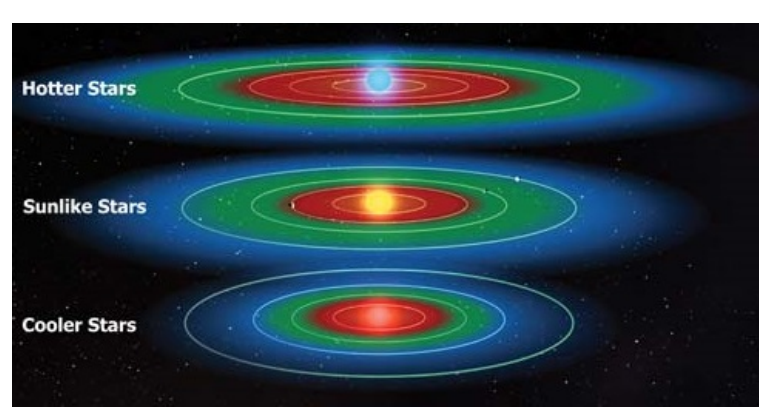

Figure 2: The three reprehensive star spectral types with their three zones: Hot (red colored), Habitable (green colored), Cold (blue colored).

Figure 2 is displaying a graph of the habitable zone, where in the horizontal axis are the distances in Astronomical units (distance between Sun-Earth $1 \mathrm{AU}=150 \mathrm{mil} \mathrm{km}$ ) and in the vertical axis, on the left the star masses (Mass of=Sun's mass), on the right the respective star's spectral types, which are proportional to their temperatures. The diagram includes our Solar System, where we observe that only the 3rd planet-Earth- is inside the habitable zone. The dotted slant strait line is showing the distance where the planets are tidally locked, showing permanently the one hemisphere to their parent star. Here, beware, that planet Mercury is inside (left) this radius, but it's not locked. (Still, at least). It is rotating around its axis every 58 Earth days. The theory is saying that in every planetary, and satellite system the tidal distortions by gravitational forces on their bodies are braking their rotation by a strength inversely proportional to their distances from their central massive body (either a star, or a giant planet). The result from this theory is that all planets, and satellites, sometime in distant future, will be tidally locked with sequence from the closest one to the farthest one, maybe after hundreds of billions of years. Actually this time interval is unknown.

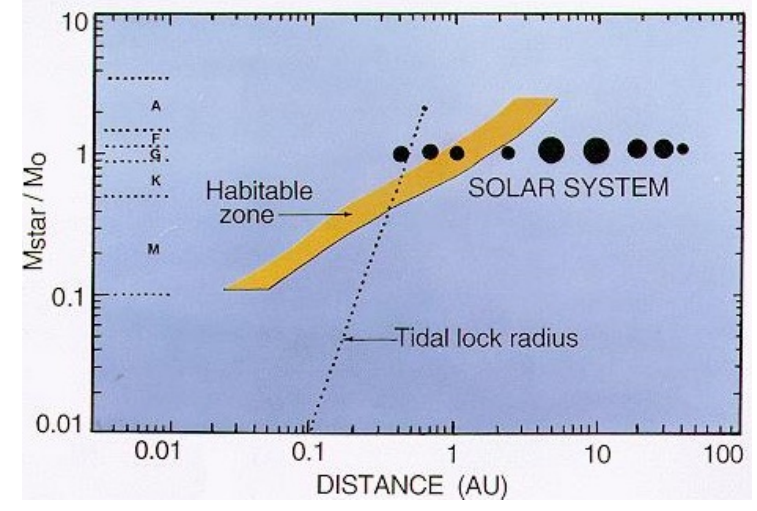

Figure 3: Notice the distribution area of habitable zone among star spectral types on the left vertical axis, as it combines with tidal lock radius.
For our Earth was found, according to geological discovering, that on the beginning of it's formation it was rotating once per 17 hours, not 24 as today. Maybe this slows down happened after collisions with planetisimals during the violent era on the early Solar System [2].

After all that, in Figure 3 the tidal lock radius was drawn empirically, without mathematical calculations based on statistics by the multitude of extra solar planets tidally locked on their parent stars.

After the above descriptions of the extra solar planetary systems, let's have a detailed look at the adequacy of the habitable zone to concentrate our research on the existence of appropriate conditions for extra terrestrial life, according to the title of this article.

As we will see below, things until now are not at all simple.

The numbers of exoplanetary systems which are already confirmed, more than 2.500, as we mentioned at the beginning, were enough to come up with a first conclusion, which is anything but optimistic [3].

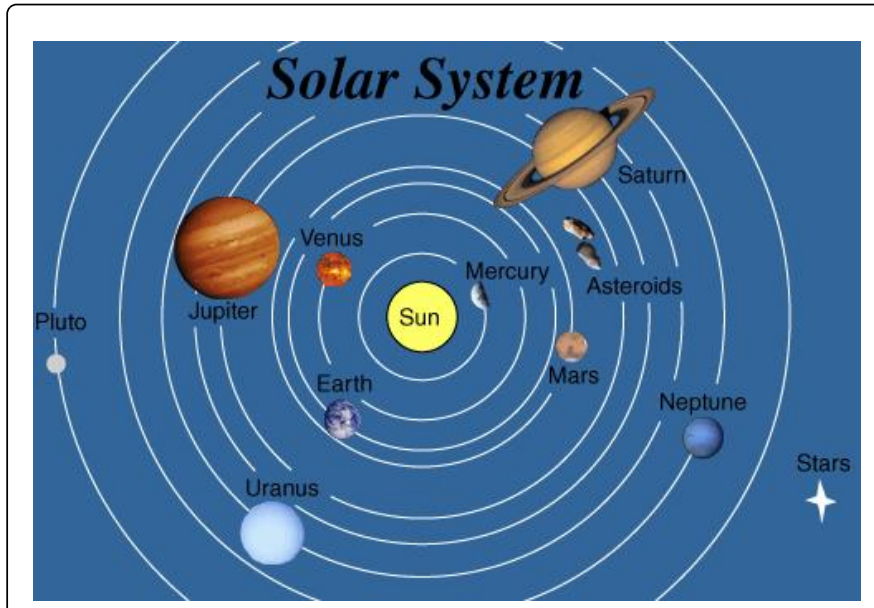

Figure 4: The shapes and distances of planet orbits are proportional with the real respective.

In our own Solar System (Figure 4) we take it for granted that all 8 planets are orbiting the Sun in the same direction, in ellipses, which are differing inconsiderably from a cycle. In mathematical- Geometric descriptions, we can say that the ellipses have their two focal points very close to each other; the one collocates (coincides) with the center of the Sun, and the other is located inside the Sun, very close to its core. The planets orbiting planes do not exceed $10^{\circ}$ among them and their intersection with ecliptic plane is spread evenly. Even dwarf planets such as Pluto and the others (Eris, Disnomia, Sedna etc) in the Kuiper belt are orbiting in the same direction with somewhat more eccentric and somewhat non concentered ellipses, with Pluto's orbital plane to intersect $17^{\circ}$ the ecliptic respective [4].

All these, consist a detailed definition of a stable and regular planetary system, our Solar System with its 3D Planet Earth, inside its habitable zone, to be inhabited by us.

On the other hand, as long as the scientists are raising the number of exoplanetary systems, which they observe and confirming, they are realizing more and more bizarre situations and conditions so that the above stability and regularity of our Solar System pass into the realm of exception rather than the rule. 
Page 3 of 6

Let's have a look at some extreme bizarre conditions on specific confirmed observations of exoplanetary systems:

The gas giant 2MASS J2126-8140 is orbiting its parent star, a red dwarf TYC 9486-9271, once every 900.000 years. Is being considered the contingency this gas giant used to be a rogue (free floating) planet, at some point approached the above red dwarf, captured from its gravitational field and started orbiting at this huge distance. Its mass is 13 Mass of (Mass of $=1$ Jupiter mass) and may be consider as a brown dwarf. These are denoting similarities with Proxima Centauri (4.3 light years) a red dwarf, which is orbiting binary star system a Centauri once every 1 million years.

Planet Kepler $432 \mathrm{~b}$ is grazing its parent star, a variable red giant which is a dying star in a minimum distance of 24 million $\mathrm{km}$ and a maximum at 54 million $\mathrm{km}$. It's estimated that after 200 million years it'll be merged in this red giant and will be vaporized.

Another planet SWEEPS 10 is orbiting its parent star, a red dwarf with mass the half of Solar mass, J175902.00-291323.7, 22 light years from here, once every 10 days, in a distance just 1.2 million $\mathrm{km}$.

The planet, which is bearing as a comet, Planet HD 20782b a gas giant with a mass twice as Jupiter's is orbiting its parent star- like our Sun spectral type G-in an orbit which is a narrow ellipse and it's minimum inside distance is 9 million $\mathrm{km}$, and a maximum of 400 million $\mathrm{km}$, which is the distance of the asteroid belt in our Solar System. When this planet is moving towards the star from maximum to minimum distance its motion is accelerating against stellar wind, and the planet's atmosphere is blasting away to the other side of the planet's motion, leaving a tail similar (visually at least) to a comet. Here we have to point out, that there is a suspicion that this accelerating speed as the planet is moving towards the star, is caused by gravitational attraction of another, more massive gas giant or a brown dwarf making the star HD 20782 to be a binary system. Until now, none of the above has been detected [5].

Gravitational interactions between two or more planets, mostly gas giants with strong gravitational fields, were observed in some exoplanetary systems, to have approached considerably one another, resulting to hurtle the one being very close to its parent star, and the other at the upper limits of its planetary system. These representative planetary systems are CVSO 30, a red dwarf star, where planet CVSO $30 \mathrm{~b}$ is orbiting just 1.2 million $\mathrm{km}$ and CVSO $30 \mathrm{c}$ at 99 billion $\mathrm{km}$, spending 27,000 years for one orbit. Other cases which have been observed are collisions between planets, or also spiral orbits which are converging towards their star, covering their Roche lobe which is shrinking due to short distance, and starting to rip, and its mass to flow on its star. This case is exoplanet WASP-18b.

The Backwards Planets: The case of exoplanet HAT-P-7b, which is a gas giant. Its parent star has a companion, with its orbital plane intersecting the ecliptic plane in a wide angle. Sometime this companion passed close by planet HAT-P-7b. Their combined motion caused the gravitational interaction between them to reverse the orbital direction of the planet. Until now eight exoplanets have detected with reverse orbital direction.

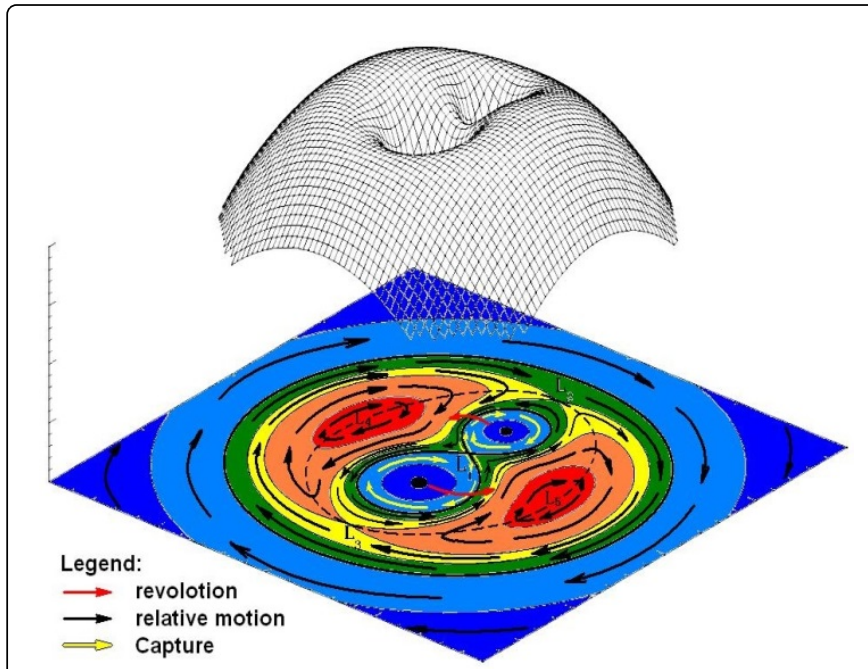

Figure 5: Whichever material body will follows the arrows direction. The centered red arrows from star members of a binary system are showing their motion around their common center of gravity.

Now, and to avoid prolonging, let's have a general look at what is going on with detected exoplanets in binary, triple, or multiple star systems. In a big percentage among them, the case, which has prevailed, is that every member of a star system has its own planets. However it depends on the average distance among these members. The factors which are involving on planets orbits are many, unpredictable, and chaotically complicated, as e.g. stars-member's mass differences, distances as previously noticed, different star evolution stages etc. We can still say, it's possible that planets-exchanges or transactions among stars-members are taking place like in banks which are the Roche Lobes (Figure 5).

That means when a planet's orbit overlaps its parent-star's Roche lobe the planet continues its orbit on the other star-member of the system. A glimpse on Figure 5, the arrows directions are enough to perceive what is going on there. The only case, which gives possibilities of normality on planets orbits, is stars-members to be close enough, so that the planets motion is able to be on the last fat blue zone on Figure 5. But it is most likely, this zone to be beyond the combined habitable zone of the stars, which depends on stars combined spectral type, and which is variably distorted due to the stars motion around their common center of gravity.

After this description of this sample of extreme cases of exoplanetary systems, I think it is unnecessary to say something about small rocky planets, (Earths, Super Earths) which may exists in these kinds of exoplanetary system, and what would be their fate over there. Thinking about our main theme, which is the habitable zones, I think that in these violent conditions, they completely lose their value, and any existing Earth like planets will come in and out of habitable zones during their relatively few years (always in astronomical scaling) of their survival.

What conclusions can we draw from the above chaotic and violent events? Why are they happening there and not here in our Solar System? The answer is below in the definition of causing chaotic situations [6]. 
Page 4 of 6

\section{In an orderly system a small interactive disorder is able to procure chaos}

Actually our Solar system is in orderly. Until when? It's not known by what, from where, and when, will chaotic conditions procured.

The above definition is applicable in all celestial systems and formations, from planetary systems, star clusters galaxies and eventually in the entire Universe. It has to do with the increasing entropy and the arrow of time as we are experiencing it.

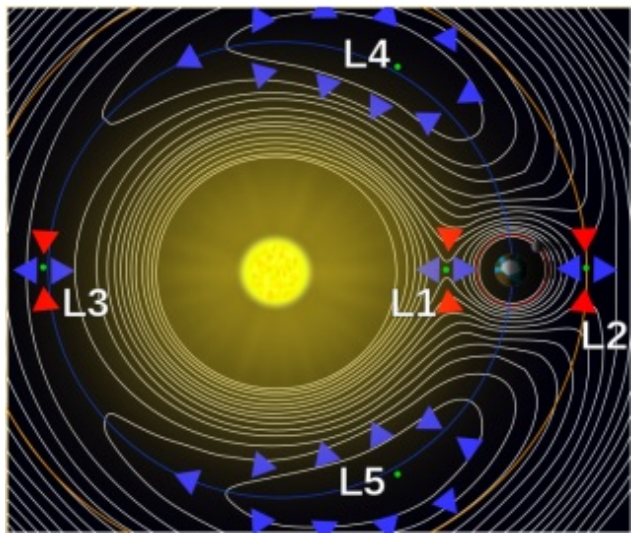

Figure 6: Sun-Earth system where are depicted distribution of equal gravitational curves and respective Roche lobes.

We can have a look to obtain a sense of our Solar System orderliness from Figure 6 where we see the almost concentric distribution of curves with equal gravitational forces between the Sun-Earth system, where are distinguished the Roche lobes, and the 5 Lagrangian points. The whole imaging as it looks; we can see that it is able to maintain orderliness and regularity in our Solar System. The other planets, are maintaining sufficient distances so that the concentricity of curves with equal gravitational force, extends beyond Lagrangian points distortions. In the past, at the beginning of our Solar System, prevailed very violent chaotic conditions, as protoplanets were shuffling with many (Apr. 80) colliding planetisimals among them, and bombardment with meteors and asteroids, an era, which is called Heavy bombardment (The predominant theory of the Moon's formation, after a collision of the proto-Earth with a body-planetisimal of the size of Mars). Additionally orbit migrations were taking place etc.

Actually as Scientists are observing planetary systems on these chaotic conditions they are working hard to be led to their past in order to unmask how and why in a specific planetary system prevailed these chaotic orbital conditions of its planets, and also how and why it is feasible in a planetary system, including our Solar System, to evolve from chaotic conditions in orderly.

These efforts based upon statistics, led to the conclusion that the gas giants are the ones, which regulate the orderliness or the chaotic conditions in a planetary system.

Thus the scientists have to focus their research on the past of planetary systems via statistics from observations of various star ages and their planets, to shed some light on the history of our own solar system, through the orbital history of Jupiter and Saturn, especially Jupiter, whose mass exceeds the sum of the rest of the Solar System's 7 planets' masses, as well as what intervenes, and prevails this normality so we are living here on Earth, and read now these lines.

Simulations in powerful computer clusters show what we have to know about where Jupiter was formed during the formation of Solar System. Actually NASA had predicted for Juno mission to be programmed to research whether Jupiter has formed elsewhere, either close to the Sun, or farther out of it, and later migrated to its present orbital position. Observational statistics are showing that such migrations happened probably at the beginning, after the formation of planetary systems. Observations of several extra solar planetary systems are unfolding in front of us several stages; from new-born, to old exoplanetary systems, up to much older than our Solar System. Kepler 444 is aged 11 billion years old. This means 6.6 billion years older than our Solar System.

Juno will be measuring water vapor in the Jovian atmosphere and the abundance of oxygen too. Oxygen is thought to correlate with the initial position from which Jupiter originated and also, many others Jovian details (which are analyzed in respective source on the last page).

Let's leave now the above chaotic conditions and come back to organized and regular planetary systems like our Solar System, and research what is going on in the habitable zones of 3 sampled star spectral types, which are imaged in Figure 2. We will bypass sunlike stars, as the conditions in our Solar System are known. However this does not mean that in other exoplanetary systems in other sunlike stars, the conditions will be the same; simply we don't know, or we cannot imagine.

We start our research at cooler stars especially red dwarfs which are the most populated and most long lived kind of stars among galaxies in the Universe. It is not accidental that the most exoplanets were detected at this kind of stars. Reasonably we can consider that based on these above properties the exoplanets in their habitable zones could be habited even by highly advanced civilizations, more possibly than any other spectral type of stars; having available all time to reach implausible levels of evolution.

However the astrophysical facts, as we'll develop now, are not showing easy and comfortable conditions of the above habitability.

The red dwarfs as stars, with a mass lower than the Sun's, are producing energy in lower rates than the Sun's rates. The result is, the transporting energy from its production area, in the star's core by thermonuclear reaction, commutating hydrogen to helium by protonproton reactions (as in our Sun), up to its imaginative surfacephotosphere. However, there is a difference in the way of transporting energy. So: in our Sun the energy is transported from its core up to almost $3 / 4^{\text {th }}$ of the way to photosphere, by radiation pressure, and in the rest is up to the surface by convection currents which are producing the magnetic fields responsible for the entire Sun's activity, sunspots, solar flares coronal mass ejections etc. On the contrary, in a red dwarf the respective radiation pressure is too weak, resulting that the energy transportation is done by convection currents in the star's entire mass so, the stirring of almost most material of the star's mass, produces enormously powerful magnetic fields so that the formation of huge star spots occur, which are increasing or decreasing it's luminosity up to $30 \%$ to $40 \%$. Also huge CMEs are created and its star flares are chucking in volumes of hot plasma, and due to star's low gravity are achieving breakneck speeds of many millions $\mathrm{km} / \mathrm{h}$. In this spectral type stars are belonging to a special kind named "flare stars", with giant 
Page 5 of 6

flares, which are able to duplicate the star's luminosity in a few minutes, lasting approximately one hour.

After that we could say that the red dwarf's habitable zone loses its meaning and its habitability, due to close proximity to the star.

Any planets in the habitable zone would be tidally locked, according to Figure 3. The hemisphere, permanently tied to the star, will be receiving mercilessly these disastrous phenomena, which are able to affect the whole planet.

There is a possibility of life growth deep inside oceans.

As for high spectral types of stars, I have in mind, that the scientific community has not shown much interest for exoplanets' existence there, due to their powerful luminosity, which maybe is a barrier to their detection. But whatever has more importance is the possibilities for a life growth are negligible up to zeroing. The main reason is that these types of stars are short-lived from some decades up to a few hundred million years.

A rocky planet, inside its habitable zone, would lashed by powerful star winds from its parent star, which is able to reach a speed up to 35 million $\mathrm{km} / \mathrm{h}$. Even if the planet has a powerful magnetic field, it would not be able to deflect the wind's particles. Gradually the planet will be stripped off of its atmosphere in space, and will be followed by evaporation of its oceans evaporation [7].

\section{Epilogue}

What we have developed upto now shows the steps scientists are following, to focus research for the conditions developing extra terrestrial life. We should consider the habitable zones as origin points in our research, without being satisfied with them.

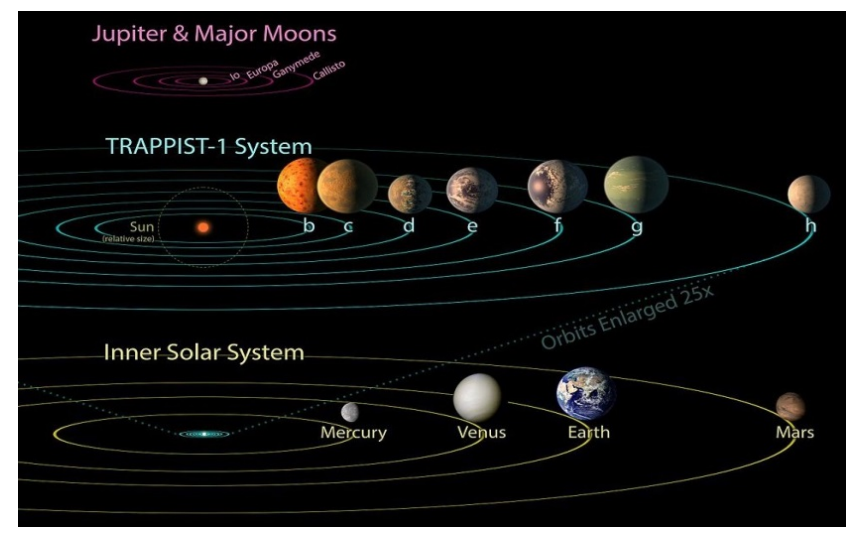

Figure 7: The Trappist 1 system is comparing not only with our own inner Solar System, but also with Jupiter's 4 major satellites. The dim dotted cycle around red dwarf Trappist 1 is the relative size of Sun.

The unfolded methodology in this article should set as the first consideration the proportion/stability? Statistics which is expected from the continuously rising number of detected exoplanetary systems, 2.588 as we mentioned at the beginning; the number among them which are observed and evaluated in orderly are only 92 .

This evaluation however is based upon the "few" years of exoplanetary astronomy nativity, which is nothing in macro world events progress timeline. Let's have a look at recently discovered exoplanetary system, Trappist 1, about which, has been said so much. It looks to be in orderliness. A glimpse at Figure 7, I think will be enough to make us ask until when?? This proximity of all its planets is able to distort the concentricity of isogravitational curves (Figure 6) and mixing up Lagrangian points' distortions. These will constitute this small interactive disorder which will convert the order into disorder after a few millions of years.

With the aim of distinguishing these exoplanetary systems, which are able to maintain their orderliness for a sufficient timeline so to evolve life, the detection of overall number of exoplanets in an exoplanetary system is necessary. In other words, a completeness of as many as possible exoplanetary systems, by the next generation of space, and ground based observatories, as the "Hi-Tech" space observatories, TESS (Transiting Exoplanet Survey Satellite), James Webb Space Telescope, W.F.I.R.S.T. (Wide-Field Infrared Survey Telescope). That, the proper software will be installed on them, so that they'll be linked together, and they'll be sending data about the exoplanet's climatic-meteorological conditions. After that the previously mentioned number 92 will be changed.

\section{Conclusion}

Beyond the research in habitable zones of stars aiming to find exoplanets similar to Earth, it would be good to keep in mind the frozen blue areas, where the existence of life in different conditions is possible. Living organisms are able to adjust themselves in environments where they are born; even in extreme environmental conditions. The variety of life here on Earth has already shown us that this is possible. Outside of Earth, in our neighborhood, in our Solar System there are possibilities of life existence in warm oceans below the thick ice layer on Jupiter's, and Saturn's satellites Europa, Callisto, Ganymede, Enceladus, and Titan; even in Pluto, according to data sent by the spacecraft "New Horizons". So why should not happen the same in exoplanets and exosatetllites around gas giants, blue zones beyond the habitable respective? The unknown " $x$ " is that it's not possible to know the evolution level of any kind of life which maybe exists over there. Currently let's be satisfied with NASA's planned mission to Jupiter's Europa [8].

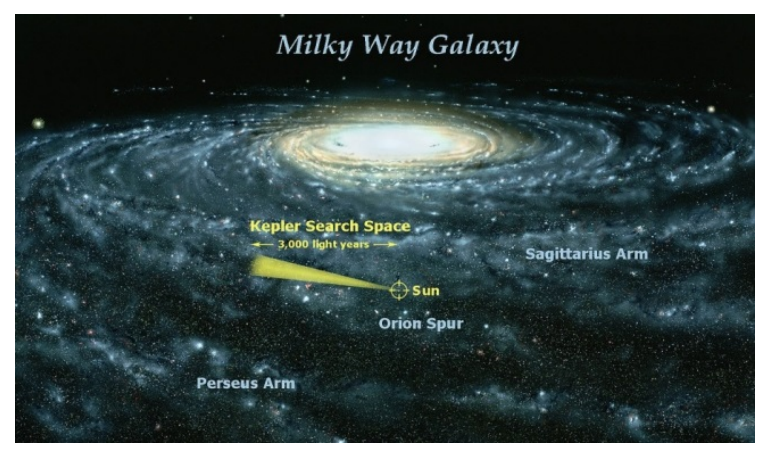

Figure 8: Kepler's search space is so tiny comparing in the huge extension of Milky Way.

But we should not be disappointed. In Figure 8 is depicted the conical sector of Kepler space observatory planet hunting, where the most exoplanets, and exoplanetary systems have been detected, in comparison with our entire Milky Way extent. According to this comparison, the scientific society has its statistic data ready, which are showing that the number of rocky planets, i.e. Earths and Super Earths probably reaches 12 billion, and we can conclude that, there will be a 
very large multiple of statistical $\mathrm{Nr} 92$ of stable and orderly planetary systems including our Solar System.

\section{References}

1. Ian S (1997) Does God play dice? The new mathematics of chaos Penguin UK.

2. Pickover C (1998) The science of aliens. Questia.

3. http://www.bizsiziz.com/the-wild-wild-worlds-a-guide-to-the-weirdestplanets-in-the-milky-way/
4. https://www.youtube.com/watch?v=uq14s5-FKhc

5. https://en.wikipedia.org/wiki/Roche_lobe

6. https://el.wikipedia.org/wiki/Red_dwarf

7. https://en.wikipedia.org/wiki/Entropy

8. http://www.nasa.gov/feature/jpl/jupiters-extended-family-a-billion-ormore 